\title{
Enquête
}

Archives de la revue Enquête

$4 \mid 1996$

La ville des sciences sociales

\section{Mesurer les mobilités urbaines}

Strasbourg 1870-1940 : trajectoires individuelles et espace urbain

Measuring urban mobilities. Strasburg 1870-1940 : individual trajectories and urban space

Jean-Luc Pinol

\section{OpenEdition}

Journals

\section{Édition électronique}

URL : http://journals.openedition.org/enquete/753

DOI : 10.4000/enquete.753

ISSN : 1953-809X

\section{Éditeur :}

Cercom, Éditions Parenthèses

\section{Édition imprimée}

Date de publication : 1 novembre 1996

Pagination : 93-106

\section{Référence électronique}

Jean-Luc Pinol, « Mesurer les mobilités urbaines », Enquête [En ligne], 4 | 1996, mis en ligne le 11 juillet 2013, consulté le 19 avril 2019. URL : http://journals.openedition.org/enquete/753 ; DOI : 10.4000/ enquete.753

Ce document a été généré automatiquement le 19 avril 2019. 


\title{
Mesurer les mobilités urbaines
}

\author{
Strasbourg 1870-1940 : trajectoires individuelles et espace urbain \\ Measuring urban mobilities. Strasburg 1870-1940 : individual trajectories and \\ urban space
}

Jean-Luc Pinol

Que signifie l'étude d'une société urbaine ou d'un morceau de ville? Peut-on choisir d'étudier un quartier sans s'interroger sur la manière dont il intervient dans le système de la ville ? Peut-on comprendre les rapports d'un groupe et de l'espace urbain en faisant abstraction du rapport que ce groupe entretient avec les autres composantes de la société urbaine? Autant de questions qu'il importe de se poser avant de se lancer à corps perdu dans le dépouillement de liasses et de liasses d'archives. À ces questions s'en ajoutent d'autres, tout aussi essentielles, qui renvoient au temps et à la perspective retenue pour lire une société urbaine. Ces questions peuvent se résumer en une seule: dresser le tableau d'une société urbaine, à un moment donné, a-t-il un sens ? La question peut être légitimement posée. Que fait-on lorsque l'on procède de la sorte? Faire une coupe transversale dans une société urbaine, voire plusieurs, n'est-ce pas s'interdire de comprendre la complexité de son fonctionnement puisque, par construction, on s'interdit d'analyser les mobilités des individus qui composent cette société ?

2 Pourtant, nombre d'études sur les villes ou sur des «morceaux de ville » fonctionnent selon ces principes. Après avoir délimité son terrain en fonction de limites administratives ou morphologiques - très souvent au niveau de ce que l'on dénomme, faute de mieux, un quartier - le chercheur s'attache à décrire les relations qui régissent les groupes sociaux entre eux, à comprendre les rapports entre parc immobilier et habitants ${ }^{1}$. Telle est la démarche que suit l'une des meilleures études sur une ville française du XIX ${ }^{e}$ siècle, celle de William H. Sewell sur les hommes et les femmes de Marseille ${ }^{2}$. L'auteur utilise conjointement les actes de mariage et les listes nominatives des recensements pour dresser trois tableaux de la société du grand port méditerranéen, sous la Restauration, sous la seconde République et à la fin du second Empire. La combinaison des sources lui permet de construire des indices d'ouverture et de fermeture des groupes sociaux, d'analyser la mobilité sociale et de montrer les transformations de la 
distribution spatiale des groupes sociaux... Sans revenir sur les critiques que l'on peut émettre concernant l'utilisation des actes de mariages pour l'étude de la mobilité sociale puisque pères et fils ne sont pas examinés au même âge ${ }^{3}$, et tout en appréciant la finesse et l'ingéniosité de certaines analyses, force est de reconnaître que la méthode retenue ne permet pas une approche longitudinale des carrières individuelles et que, par conséquent, elle ne peut prendre en compte certains fonctionnements de la société urbaine.

3 La ville est un milieu en perpétuelle recomposition. Le flux des arrivées et celui des départs sont ininterrompus et les approches transversales et longitudinales d'une société urbaine ne donnent pas la même perception des réalités urbaines. Alors que les premières valorisent la stabilité des structures urbaines, les secondes mettent en lumière les évolutions. La permanente recomposition de la ville, et plus particulièrement de la grande ville, ne peut pas véritablement être mise en évidence par l'analyse d'une succession de coupes transversales. Surtout, ce type d'analyse interdit de comprendre les rythmes de la recomposition elle-même, faute de reconstituer les trajectoires des urbains.

La méthode longitudinale est délicate car les sources permettant de manière immédiate ce type d'approche sont rares. Les plus adéquates sont celles qui relèvent de la logique des registres de population, mais il est aussi possible de reconstituer les itinéraires des citadins à partir de documents qui échappent à cette logique. Avant d'examiner les résultats obtenus à partir de registres de population, rappelons brièvement les exigences d'une telle démarche. Pour une très grande ville, Lyon, j'ai utilisé les listes électorales, qui ont le grand mérite d'être classées par ordre alphabétique, pour reconstituer deux cohortes de Lyonnais, la première née au début de la Troisième République, la seconde née au tournant du siècle. La reconstitution a porté sur près de 1600 électeurs, les 800 premiers ont été suivis pendant quarante ans, de 1896 à 1936, les 800 autres pendant quinze ans, de 1921 à 1936. Ont été mis en évidence les effets d'âge et les phénomènes de génération dans les domaines de la mobilité résidentielle, des mobilités intra- et intergénérationnelles ${ }^{4}$. Pour des villes de taille plus modeste, on peut utiliser les listes nominatives du recensement, en reconstituant à partir de documents présentant une photographie à un moment donné, le film des itinéraires individuels. C'est de cette manière que A.Girot a étudié la mobilité des habitants d'une petite ville textile des Vosges ${ }^{5}$. Elle a reconstitué les itinéraires de tous les chefs de ménage dont le patronyme commence par la lettre B entre 1881 et 1926. Une telle étude impose la lecture exhaustive des listes nominatives; elle est donc longue et ne peut être mise en œuvre que pour des villes de taille modeste. Tel est le cas de Moyenmoutier qui ne dépasse pas les 2500 habitants agglomérés au chef-lieu pendant la période étudiée. Une telle opération serait difficilement concevable dans le cadre d'une grande ville comme Lyon en raison du nombre de volumes des listes nominatives ${ }^{6}$. Une différence fondamentale se dégage entre la ville textile de taille modeste, où le patronat développe une politique de contrôle social qui s'appuie, entre autres, sur la construction de logements ouvriers, et les grandes villes marquées par un renouvellement continuel de leur population.

5 Toute différente est la logique des documents dynamiques de type «registre de population » tels qu'ils existent en Belgique ${ }^{7}$, en Allemagne ${ }^{8}$ ou en Italie où les documents de l'anagrafe ont été utilisés par Olivier Faron ou Maurizio Gribaudi. Les recherches de M. Gribaudi sur Turin ${ }^{9}$ combinent histoire orale et reconstitution de trajectoires individuelles à partir de l'anagrafe. Les itinéraires d'ouvriers reconstitués portent sur cinq générations. M. Gribaudi dégage les logiques de ce qu'il nomme le cycle d'intégration 
urbaine. Parallèlement aux stratégies professionnelles se développent de véritables stratégies résidentielles et cela lui permet de distinguer ce qu'il nomme les quartiers hybrides où les ouvriers ne dépassent pas $40 \%$ de la population totale et les quartiers périphériques où ils dépassent $70 \%$. Les premiers correspondent aux quartiers anciens, où s'entremêlent une multitude de groupes sociaux et où l'hétérogénéité sociale est la règle. Les seconds, qui se développent juste avant ou juste après la Première Guerre mondiale, sont plus homogènes socialement et le monde ouvrier y est hégémonique. Celles d'O. Faron sur Milan ${ }^{10}$ obligent à repenser la manière d'écrire l'histoire sociale des villes. Plutôt que de définir des groupes sociaux dont on analyse l'homogénéité des comportements, l'anagrafe permet de décrire les comportements des individus, de lire les pratiques et donc de retrouver de véritables projets individuels ou familiaux. Parmi les comportements lentement mûris, O.Faron, attache une place particulièrement importante à la limitation de la taille des familles. Cette limitation ne correspond pas à un simple ajustement de la démographie à la conjoncture économique mais renvoie à un véritable « choix socio-culturel » des citadins.

6 Les recherches en cours de René Lorenceau sur les archives de Bâle montrent la pertinence d'une démarche fondée sur une batterie de documents inspirés de la logique des registres de population. Différents registres destinés à contrôler les résidents permanents et les résidents temporaires et leur éventuel accès à la bourgeoisie autorisent l'étude des processus d'intégration des nouveaux venus ${ }^{11}$, ils permettent aussi d'étudier leurs trajectoires urbaines et de distinguer les différences qui apparaissent d'une génération à l'autre entre les années 1850 et la Première Guerre mondiale ${ }^{12}$. C'est dans le même esprit qu'ont été entreprises les recherches sur les registres de Strasbourg, soit dans le cadre de recherches individuelles, soit dans celui de deux contrats de recherches.

7 Le fichier domiciliaire de Strasbourg ne distingue pas comme celui de Bâle ou de Colmar ${ }^{13}$ les habitants en fonction de leur statut ou de leur nationalité : tous les habitants figurent dans un seul et même fichier mis en place par l'administration allemande après $1871^{14}$. Dès septembre 1871, le directeur de la police de Strasbourg informait la population de l'ouverture d'un bureau des déclarations domiciliaires. Son fonctionnement est explicité par une ordonnance du président de district (Bezirkpräsident) qui contrôle l'administration communale en date du 16 juin $1883^{15}$. Le droit pénal français faisait obligation aux aubergistes et aux loueurs de pièces d'habitation et de garnis de déclarer à la police municipale l'arrivée et le départ de locataires ou de clients. L'ordonnance du 16 juin 1883 confirme cette obligation et l'étend à l'ensemble de la population. Les infractions étaient sanctionnées. Après novembre 1918, le droit français se combine au droit local et la pratique administrative admet que l'ordonnance de juin 1883 reste en vigueur: dans le Strasbourg de l'entre-deux-guerres, les habitants achètent dans les bureaux de tabac les formulaires nécessaires à la déclaration d'arrivée ou de départ d'un nouveau logement ${ }^{16}$.

8 Les recherches ont été menées en deux temps. Le premier temps a porté sur une population prédéfinie pour laquelle une liste d'individus permettait une utilisation simple du fichier domiciliaire. Dans un deuxième temps, un échantillon représentatif a été constitué.

9 Les résultats de ces enquêtes obligent à repenser le fonctionnement d'une grande ville. À lui seul, le nombre de chefs de ménage répertoriés - près de 1200000 fiches individuelles - indique l'importance de la mobilité pour une ville dont la population totale a oscillé, pendant la période 1870-1940, entre 70000 et 190000 habitants. Les fiches donnent l'ensemble des adresses occupées par le chef de ménage à Strasbourg. Lorsque le chef de 
ménage quitte la ville et y revient, le lieu de séjour en dehors de Strasbourg est fréquemment indiqué. Pour l'étude de la mobilité intra-urbaine et pour la connaissance des relations que la ville entretient avec son bassin démographique, ces registres permettent donc de répondre à de nombreuses questions que l'on se poserait en vain pour beaucoup d'autres villes.

10 Autant il est possible lorsque l'on a une liste de chefs de ménage de les rechercher dans les fichiers - et cela a permis de faire des recherches sur la manière dont s'était constituée la population de tel ou tel groupe d'immeubles en utilisant comme base de départ les listes nominatives des recensements de $1936^{17}$ ou d'étudier la mobilité de tel groupe particulier, les professions juridiques ${ }^{18}$, les boulangers, les universitaires... autant le sondage apparaissait comme la seule méthode permettant une pesée globale des mobilités.

$11 \mathrm{Au}$ lendemain du retour de l'Alsace dans le giron de la Troisième République, les universitaires allemands quittent le Reichsland et ils sont bientôt remplacés par des fonctionnaires français. Près de 270 universitaires ont exercé leur fonction à Strasbourg pendant l'entre-deux-guerres. À partir de la liste de ces universitaires, il a été possible de reconstituer leur itinéraire urbain. La question essentielle était alors celle des relations qu'un groupe social apparemment bien défini entretient avec une grande ville. La réponse est claire : le groupe social en question n'a pas d'homogénéité face à la ville. Les clivages renvoient aussi bien à l'origine géographique qu'à la religion déclarée, aussi bien à la distribution des individus dans l'espace urbain qu'à la discipline enseignée. La diversité des pratiques sociales entraîne un véritable éclatement du groupe social en tant que tel. Les professeurs de lettres, originaires de Paris ou d'autres grandes villes de France, se distinguent fondamentalement des médecins, plus enracinés dans le monde alsacien, qui eux-mêmes n'ont que peu de points communs avec des pharmaciens. Le suivi des itinéraires urbains des universitaires - le rythme même des changements résidentiels est un paramètre important - souligne le caractère partiel d'une histoire sociale de la ville qui ne prendrait pas en compte les mobilités des individus ${ }^{19}$.

Une telle approche restreinte à un nombre limité de chefs de ménage ne permet pas pour autant de mettre en lumière le fonctionnement de l'ensemble de la société urbaine. Une étude exhaustive était hors de portée, à cause de la masse documentaire à traiter, l'étude par sondage s'impose. Encore fallait-il trouver une méthode relativement économe en temps et permettant un sondage pertinent. Le choix a été fait d'étudier les chefs de ménages dont le patronyme commence par $\mathrm{N}$. Ont été microfilmées les fiches correspondant à 15300 chefs de ménages répartis ainsi entre trois fichiers chronologiques successifs: 2950 pour les années 1871-1895, 5850 pour les années 1896-1918 et 6500 pour les années 1919-1939. Une base de données a été constituée à partir de ces trois fichiers. Elle porte sur 7500 chefs de ménage (1 450 dans le premier fichier, 2865 dans le deuxième et 3185 dans le dernier) ${ }^{20}$.

13 Avant de montrer en quoi les suivis longitudinaux remettent en cause nombre de « certitudes » sur le fonctionnement des sociétés urbaines, donnons rapidement quelques résultats globaux qui soulignent l'extrême mobilité des populations urbaines. L'information la plus importante est la suivante : $40 \%$ des chefs de ménage restent dans la ville moins de six mois et la moyenne de leur séjour est inférieure à deux mois. Ce chiffre, à lui seul, invalide toutes les approches statiques d'une société urbaine. Plusieurs remarques : ce taux n'est pas homogène pendant toute la période étudiée ; alors qu'il est de l'ordre d'un tiers avant la Première Guerre mondiale, il progresse pendant la guerre et 
s'établit à $48 \%$ pendant la période de l'entre-deux-guerres. Cela signifie que pendant cette période, près d'un arrivant sur deux reste à Strasbourg moins de six mois et en moyenne moins de deux mois. Par ailleurs, la durée du séjour n'est pas fondamentalement différente entre hommes et femmes. Parmi les hommes, les trois quarts des chefs de ménage hommes sont célibataires; parmi les femmes plus de quatre sur cinq.

Entre 1871 et la veille de la Première Guerre mondiale, la ville est passée de 90000 habitants à 160000 habitants. Pendant cette période, le fort solde migratoire se conjugue avec une forte croissance naturelle, elle-même conséquence d'une natalité élevée. Avec la période de la guerre, il est clair que Strasbourg enregistre une nette augmentation des flux migratoires. Le solde n'est que la part émergée des flux migratoires mais il est rare que l'on puisse mesurer la relation qui existe entre les deux phénomènes. Avant les années dix, il apparaît que le rapport entre le solde et les flux est de 1 à 5 ; pendant la guerre et après, ce rapport passe à 1 ou 2 pour 100.

La grande ville apparait comme le lieu par excellence d'un intense brassage de populations. Par ailleurs, les échanges entre la ville et l'extérieur ne sont évidemment pas à sens unique : si entre 1870 et 1920, Paris fournit $3 \%$ des nouveaux arrivants, Metz, Berlin ou Mulhouse quelque $2 \%$, Colmar, Nancy, Bâle ou Cologne environ $1 \%$, ce sont plus de $5 \%$ des départs renseignés qui s'effectuent vers Paris, $2 \%$ environ vers Berlin, Metz, Colmar, Mulhouse, $1 \%$ vers Nancy, Bâle ou Cologne...

La population des chefs de ménage qui arrivent à Strasbourg entre 1870 et 1940 est née, pour l'essentiel, en Alsace (près de $40 \%$ ), en Allemagne (quelques points de moins), en France, en Lorraine. Les régions d'origine et de destination ne sont guère différentes des régions de naissance ${ }^{21}$. Mais si l'Alsace tient la première place, il apparaît clairement que Strasbourg est un carrefour de l'Europe. En fait pendant l'entre-deux-guerres, et même dès le début de la Première Guerre mondiale, la ville fonctionne comme un sismographe des migrations venant de l'est de l'Europe.

Pour préciser les rapports qui existent entre population enracinée et oiseaux de passage, nous avons reconstitué, à partir de cette source longitudinale, deux coupes transversales qui autorisent une comparaison entre la situation de la capitale du Reichsland et la préfecture de la Troisième République. Pour une première approche, nous avons considéré comme enracinés les chefs de ménage dont le séjour à Strasbourg dépassait quinze ans. Un quart seulement des chefs de ménage présents le $1^{\text {er }}$ janvier 1890 sont dans ce $\operatorname{cas}^{22}$. Au $1^{\mathrm{er}}$ janvier 1925, un peu plus d'un tiers. On pourrait d'ailleurs se demander si, dans la capitale alsacienne et dans un contexte politique mouvant, un séjour de quinze ans avant 1890 est bien équivalent à un séjour de quinze ans avant $1925^{23}$. Le poids des enracinés progresse légèrement entre les deux dates mais ces derniers sont très minoritaires dans la société de la grande ville. Ces changements des composantes démographiques de la population se doublent de changements de la structure sociale. Entre 1890 et 1925, on assiste à un gonflement des catégories centrales de la société urbaine. Les années 1890 sont celles de la construction de la nouvelle ville et l'afflux de très nombreux manuels non qualifiés employés dans le bâtiment - on repère nombre de maçons italiens - est nécessaire aux entreprises locales. Dans le même temps, Berlin envoie de très nombreux hauts fonctionnaires pour mettre en place la nouvelle administration de la capitale du Reichsland dont l'autorité s'étend sur l'Alsace et sur la Lorraine annexée. La situation est différente au milieu des années vingt : Strasbourg n'est 
plus que la préfecture du Bas-Rhin. Les hauts fonctionnaires et les manuels non qualifiés sont moins nombreux qu'au temps de la construction de la nouvelle ville.

Le suivi des itinéraires individuels et des déplacements inter-urbains des chefs de ménage permet aussi de mieux comprendre le fonctionnement de l'espace urbain et les relations entre différents quartiers ${ }^{24}$. Pendant la période considérée, l'espace urbain augmente fortement et une nouvelle ville, la Neustadt, adaptée à la fois à des impératifs de défense et aux principes de circulation des véhicules, de l'air et de la lumière sort de terre. Ce nouvel espace urbain, à la fois conçu comme lieu du pouvoir et de la culture ${ }^{25}$, devient le quartier résidentiel par excellence ${ }^{26}$. Y sont construits bâtiments officiels, immeubles et villas où vont séjourner les fonctionnaires de l'Empire. Le suivi longitudinal des chefs de ménage permet de constater en quoi son développement pèse sur les relations qui existent entre les différentes unités urbaines.

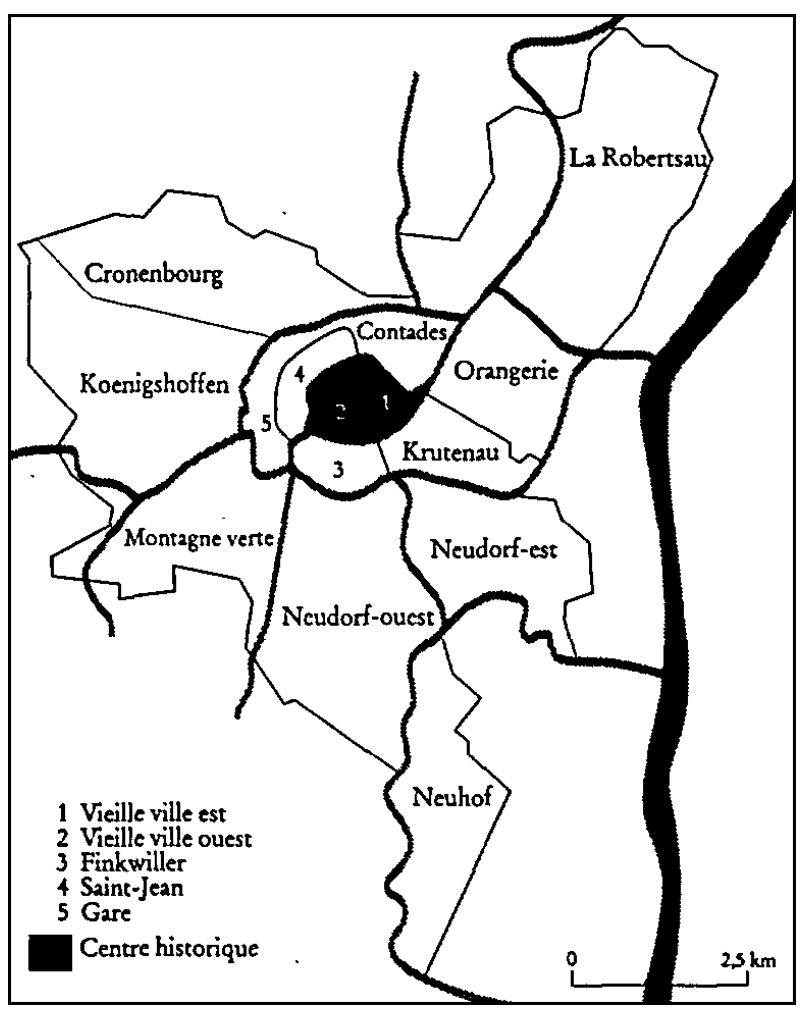

Certains quartiers ont un rôle particulier dans l'accueil de la population migrante qui ne reste que peu de temps à Strasbourg. Dans la région lyonnaise, on les appelle des oiseaux de passage; en Alsace on parle des accourus, les «hergelofene». Ces hommes et ces femmes restent moins de six mois dans la ville et leur séjour moyen est de deux mois environ. Leur distribution dans l'espace urbain est très spécifique. Les deux principaux quartiers d'accueil de ces chefs de ménage, très souvent célibataires, sont la partie ouest de la vieille ville et le quartier Saint-Jean qui la jouxte mais d'autres quartiers peuvent avoir la même fonction, au moins pour un temps. Tel est le cas des deux quartiers de la Neustadt (l'Orangerie et les Contades) avant qu'ils ne soient complètement bâtis. Avant le tournant du siècle, la présence d'ouvriers du bâtiment et de femmes de ménage explique leur classement dans cette catégorie mais dès que la ville neuve atteint un certain seuil d'occupation, elle n'apparaît plus comme une zone d'accueil des oiseaux de passage. Pendant l'entre-deux-guerres, la période où Strasbourg enregistre le turn-over maximum de sa population, la vieille ville dans son ensemble ainsi que le quartier de la gare sont le lieu de prédilection des installations temporaires. Les quartiers périphériques situés au- 
delà des fortifications sont plutôt les lieux de prédilection des chefs de ménage qui restent plus de cinq ans à Strasbourg mais là aussi, les reclassements sont fréquents. Cette façon de décrire les différents espaces de la ville n'est pas fausse, elle correspond à une situation à un moment donné, mais elle est aussi trompeuse car elle ne prend pas assez en compte la dynamique urbaine. Pour cela, il faut concentrer l'analyse sur les individus qui ont au moins deux adresses dans la ville et représentent la part la plus stable de la population de la ville ${ }^{27}$. Le tableau suivant donne, pour deux cohortes d'arrivants, la part de ceux qui ont leur première et leur seconde adresse dans le même quartier (\% de stables), la durée du séjour moyen à la première adresse (en jours) et l'effectif concerné ${ }^{28}$.

\begin{tabular}{lrrrrrr} 
& \multicolumn{2}{c}{$1890-1899$} & & $1919-1929$ & \\
\multicolumn{1}{c}{ \% de stables } & Séjour & Effectif & \% de stables & Séjour & Effectif \\
\hline Vieille ville est & 28 & 1760 & 22 & 26 & 219 & 22 \\
Vieille ville ouest & 41 & 769 & 43 & 23 & 351 & 16 \\
Saint-Jean & 36 & 811 & 34 & 28 & 298 & 19 \\
Finkwiller & 28 & 189 & 11 & 13 & 1742 & 4 \\
Krutenau & 48 & 641 & 42 & 31 & 235 & 22 \\
Orangerie & 20 & 4297 & 3 & 54 & 309 & 55 \\
Contades & 38 & 2501 & 8 & 36 & 431 & 27 \\
Gare & 26 & 250 & 6 & 20 & 262 & 9 \\
Neudorf & 46 & 2815 & 24 & 62 & 1556 & 52 \\
Neuhof & 63 & 2533 & 5 & 44 & 679 & 7 \\
Robertsau & 30 & 862 & 3 & 38 & 338 & 5 \\
Koenigshoffen & 29 & 19 & 2 & 31 & 455 & 4 \\
Montagne Verte & 63 & 640 & 5 & 18 & 2859 & 2 \\
Cronenbourg & 36 & 5620 & 4 & 33 & 124 & 5 \\
\hline & & & & & & \\
Ensemble & 38 & 947 & 212 & 36 & 611 & 249 \\
\hline
\end{tabular}

Pour l'ensemble de la ville, les variations sont assez limitées entre les deux décennies retenues : le poids moyen des stables varie peu - un peu plus du tiers des chefs de ménage a son premier et son second logement dans le même quartier - et la durée moyenne de leur résidence diminue d'environ un tiers - alors que les chefs de ménage restaient environ trente mois à leur première adresse (947 jours) entre 1890 et 1899, ils y restent vingt mois (611 jours) entre 1919 et 1929. L'explication du phénomène est à chercher dans l'importance de l'offre de logements au lendemain de la guerre, consécutive au départ massif des Allemands. Mais ce n'est pas le plus important du point de vue qui nous occupe ici : les suivis longitudinaux des chefs de ménage permettent d'analyser les reclassements des quartiers dans le système intra-urbain. Comparons les deux quartiers vieille ville situés dans l'ellipse insulaire, un quartier de la nouvelle ville, celui de l'Orangerie, et le principal quartier périphérique, Neudorf. La croissance de la banlieue sud s'explique par celle de Neudorf qui passe de moins de 5000 habitants avant la guerre de 1870 à plus de 26000 avant celle de 1914-1918. Le poids des stables, déjà important avant la fin du XIX ${ }^{\mathrm{e}}$ siècle, progresse de manière significative mais surtout les effectifs concernés sont multipliés par deux. L'enracinement d'une partie de la population ouvrière qualifiée se fait prioritairement dans la banlieue sud de la ville et il est tout à fait significatif, les effectifs concernés et la tendance confirmée d'une période d'arrivée à l'autre, que la durée du séjour initial dépasse quatre ans alors qu'elle est inférieure à un an pour les deux quartiers centraux et pour l'Orangerie. Pour la vieille ville, les différences entre les parties ouest et est sont nettes. La partie ouest, la plus taudifiée, est le théâtre de la Grande Percée, elle enregistre une chute notable des éléments stables et une chute importante de la durée de séjour. Dans la partie est, le poids des stables reste identique mais la signification même de la stabilité se modifie radicalement: le premier séjour passe de près de cinq ans à quelques mois. 
21 Les différences constatées se confirment à l'examen des flux de sortie survenus entre la première et la seconde adresse comme le montre le tableau suivant. C'est dans ce domaine que les suivis longitudinaux révèlent le mieux le fonctionnement urbain. Entre la décennie 1890-1899 et celle qui suit la Première Guerre mondiale, les quartiers qui accueillent les habitants dont la première adresse est située dans ces quatre quartiers changent, ce qui met en évidence une mutation de la stabilité résidentielle.

\begin{tabular}{|c|c|c|c|c|c|c|c|c|}
\hline \multirow{2}{*}{$\begin{array}{l}\text { Première adresse } \\
\text { Seconde adresse }\end{array}$} & \multicolumn{2}{|c|}{ Vieille Ville est } & \multicolumn{2}{|c|}{ Vieille Ville ouest } & \multicolumn{2}{|c|}{ Orangerie } & \multicolumn{2}{|c|}{ Neudorf } \\
\hline & cohorte 1 & cohorte 2 & cohorte 1 & cohorte 2 & cohorte 1 & cohorte 2 & cohorte 1 & cohorte 2 \\
\hline Vieille Ville est & 27,50 & 26,19 & 15,24 & 11,43 & 20,00 & 7,84 & 5,77 & 4,76 \\
\hline Vieille Ville ouest & 21,25 & 20,24 & 40,95 & 22,86 & 6,67 & 6,86 & 9,62 & 4,76 \\
\hline Finkwiller & 8,75 & 2,38 & 3,81 & 5,71 & 6,67 & 3,92 & 7,69 & 1,19 \\
\hline Krutenau & 7,50 & 7,14 & 9,52 & 11,43 & 20,00 & 5,88 & 11,54 & 3,57 \\
\hline Saint-Jean & 10,00 & 7,14 & 17,14 & 12,86 & 13,33 & 3,92 & 5,77 & 3,57 \\
\hline Contades & 10,00 & 13,10 & 0,95 & 14,29 & 6,67 & 11,76 & 1,92 & 1,19 \\
\hline Orangerie & 7,50 & 5,95 & 1,90 & 11,43 & 20,00 & 53,92 & 1,92 & 4,76 \\
\hline Gare & 5,00 & 4,76 & 4,76 & 1,43 & 0,00 & 1,96 & 1,92 & 2,38 \\
\hline Neudorf & 1,25 & 7,14 & 1,90 & 5,71 & 6,67 & 2,94 & 46,15 & 61,90 \\
\hline Neuhof & 0,00 & 0,00 & 0,00 & 0,00 & 0,00 & 0,00 & 1,92 & 3,57 \\
\hline M. Verte & 1,25 & 1,19 & 0,95 & 0,00 & 0,00 & 0,00 & 1,92 & 3,57 \\
\hline Koenigshoffen & 0,00 & 1,19 & 0,95 & 0,00 & 0,00 & 0,00 & 1,92 & 1,19 \\
\hline Cronenbourg & 0,00 & 0,00 & 0,95 & 1,43 & 0,00 & 0,00 & 0,00 & 1,19 \\
\hline Robertsau & 0,00 & 3,57 & 0,95 & 1,43 & 0,00 & 0,98 & 1,92 & 2,38 \\
\hline Echantillon & 80 & 84 & 105 & 70 & 15 & 102 & 52 & 84 \\
\hline
\end{tabular}

Les départs de la vieille ville vers la périphérie sud (le Neudorf) augmentent sensiblement et les flux de départs de la partie ouest et de la partie est vers la nouvelle ville sont bouleversés. Alors que les flux étaient quasi inexistants entre la partie ouest et la nouvelle ville avant la Première Guerre mondiale - la composition sociale des deux espaces était alors très contrastée -, les mutations survenues lors des travaux de la Grande Percée font des Contades le second quartier d'accueil des habitants de ce quartier après la guerre. Par ailleurs le flux en direction de l'Orangerie est multiplié par cinq.

Le tableau permet aussi de comprendre comment se construit la fermeture sur euxmêmes du nouveau quartier résidentiel et de la périphérie populaire. Seul le quartier des Contades, qui en est proche socialement et spatialement, accueille plus de $10 \%$ des habitants dont la première adresse strasbourgeoise se situe dans le quartier de l'Orangerie. Le nouveau quartier résidentiel n'enregistre quasiment aucun départ vers la périphérie. En sens inverse, à peine $5 \%$ des habitants du Neudorf ont une seconde adresse dans le nouveau quartier résidentiel. Au contraire, les échanges avec le reste de la banlieue ne sont pas rares alors que ceux avec le centre-ville ont tendance à diminuer, non seulement en pourcentage mais même en chiffres absolus alors que les effectifs pris en compte ont nettement augmenté. Là, l'approche des trajectoires urbaines permet de mettre en évidence le cloisonnement progressif du centre et de la périphérie.

L'objet de cette présentation n'était pas de décrire par le menu le fonctionnement d'une grande ville, en l'occurrence Strasbourg, mais d'utiliser une source de nature longitudinale, rarement disponible pour d'autres villes de France, afin de réfléchir, à partir des résultats obtenus, à des fonctionnements urbains qui ne sont pas propres à la seule capitale alsacienne. Si nos conclusions sont retenues, cela signifie clairement qu'une approche des sociétés urbaines qui ne se fonderait que sur une démarche transversale serait vouée, par ses présupposés mêmes, surtout s'ils ne sont pas clairement perçus par les chercheurs qui les mettent en œuvre, à orienter la réflexion vers une impasse. En effet, elle s'empêcherait de réfléchir à ce qui est l'essence même de la ville, la 
recomposition permanente de sa population. Comprendre une société urbaine implique que les individus soient saisis, à la fois, comme des éléments constitutifs des groupes sociaux mais aussi comme des individus ayant leurs trajectoires propres afin de mieux saisir la dynamique des populations urbaines. Du point de vue de l'histoire sociale, ces exigences complémentaires conduisent à élaborer une histoire sociale des individus qui associent approche transversale et approche longitudinale ${ }^{29}$.

Revenons un instant à une histoire des sociétés urbaines qui ignorerait les suivis longitudinaux. La justification du recours exclusif aux coupes transversales s'appuie fréquemment sur l'indisponibilité de sources permettant l'emploi d'une autre méthode. L'argument ne me semble pas recevable: on peut utiliser dans une perspective longitudinale des sources qui ne relèvent pas de cette perspective. Surtout, il me semble aujourd'hui impossible d'ignorer les mobilités qui caractérisent les populations urbaines: toute analyse qui se fonderait uniquement sur une approche transversale, et donc statique, risquerait d'arriver à des conclusions controuvées.

\section{NOTES}

1. L'objet de cet article reprend en partie des débats évoqués dans un numéro de Histoire, économie \& société, XIII, 1994, intitulé Lectures de la ville (XV siècle-XX ${ }^{e}$ siècle), préparé par J. Bottin et A. Cabantous. Voir en particulier les articles de J.-P. Burdy, «La monographie de quartier en histoire urbaine, quelques éléments de bilan sur une recherche stéphanoise », et de J.-L. Pinol, « Lectures transversales et longitudinales d'une société urbaine, Lyon aux XIX et $\mathrm{XX}^{\mathrm{e}}$ siècles ».

2. W. H. Sewell Jr., Structure and Mobility. The Men and Women of Marseille, 1820-1870, CambridgeParis, Cambridge University Press/Éd. de la MSH, 1985.

3. Sur ce point, voir G. Dupeux, "L'étude de la mobilité sociale, quelques problèmes de méthode", in Conjoncture économique, structures sociales. Hommage à Ernest Labrousse, Paris, Mouton, 1974, p. 79-90.

4. Voir J.-L. Pinol, Les mobilités de la grande ville. Lyon fin XIXe-début $X X^{e}$ siècle, Paris, Presses de la Fondation nationale des Sciences politiques, 1991.

5. A. Girot, Moyenmoutier, ville de mono-industrie textile entre 1880-1930, mémoire de maîtrise, Université de Strasbourg II, 1995, ms.

6. II faudrait de plus pour Lyon tenir compte de la falsification de ces listes nominatives. Sur ce point voir J. Bienfait, "La population de Lyon à travers un quart de siècle de recensements douteux, 1911-1936 ", Revue de Géographie de Lyon, XLIII, 1968, p. 63-132. Voir aussi J.-L. Pinol, Les mobilités de la grande ville..., op. cit., p. 29, 44-46, 105-106. Prendre en compte les chefs de ménage dont le patronyme commence par B - on sait que ces patronymes représentent $14 \%$ à $15 \%$ des patronymes, ibid., p. 389 - reviendrait à sélectionner tous les cinq ans quelque 14000 chefs de ménage parmi une masse de 400 à 500000 individus.

7. Pour un exemple d'utilisation des registres de Belgique, voir S. Pasleau, "L'immigration des travailleurs à Seraing durant la seconde moitié du XIX e siècle ", Annales de Démographie historique, 1993, p. 227-249.

8. Cf., par exemple, S. Leiner, Migration und Urbanisierung, Binnenwenderugsbewegungen, raümlicher und sozialer Wandel in den Industriestädten des Saar-Lor-Lux-Raumes, 1856-1910, Sarrebruck, 
Saarbrücker Druckerei und Verlag, 1994. L'étude de Stefan Leiner porte sur Esch-sur-Alzette, Thionville et Malstatt-Burbach (un quartier de Sarrebruck).

9. M. Gribaudi, Itinéraires ouvriers, Espaces et groupes sociaux à Turin au début du XXe siècle, Paris, Éd. de l'EHESS, 1987.

10. Cf. O. Faron, «L" "anagrafe" milanaise de Napoléon à l'Unité. Une source originale pour l'histoire sociale urbaine ", Mélanges de l'École française de Rome, Moyen Âge-Temps modernes, XCVIII, 1986, p. 1153-1176, et surtout sa thèse, La ville des destins croisés. Recherches sur la société milanaise du XIX ${ }^{e}$ siècle (1811-1860), Paris, EHESS, 1996, ms.

11. R. Lorenceau, Bâle 1870-1920. Croissance urbaine, mutations économiques et mobilité sociale, mémoire de DEA, Université de Strasbourg II, 1993, ms.

12. Cf. R. Lorenceau, "Statuts et changements de statuts des immigrants de la ville de Bâle, 1850-1920 », in D. Menjot et J.-L. Pinol, eds, Les immigrants et la ville, Paris, L'Harmattan, 1996.

13. À Colmar, par exemple, les Italiens sont classés dans un fichier spécial.

14. II serait faux, toutefois, de faire d'un tel document le résultat d'une pratique administrative du deuxième Reich. Dès 1791, les autorités françaises avaient envisagé une remise à jour périodique du rôle de la population - le code de police municipale de 1791 précise : «Les corps municipaux feront constater l'état des habitants [...] chaque année [...] cet état sera vérifié de nouveau, et on y fera les changements nécessaires » - mais si ces mesures ont été peu suivies en France même, elles ont été plus souvent mises en œuvre dans les territoires annexés par l'Empire (cf. O. Faron, La ville des destins croisés, op. cit., p. 27-30). Les provinces les plus récemment intégrées au Royaume, comme l'Alsace, ont-elles été plus surveillées que les autres? Cela expliquerait que dès le début $\mathrm{du} \mathrm{XIX}^{\mathrm{e}}$ siècle, des documents organisés selon le principe du registre de population existent à Strasbourg. Ils sont composés d'un index des habitants avec renvois aux fichiers adresses, ces derniers indiquant les dates d'entrée et de sortie des logements.

15. Cf. T. Duclerc, Des mobilités à Strasbourg, 1871-1939. Enquêtes et réflexions sur le fichier domiciliaire de la population strasbourgeoise, DEA, Université de Strasbourg II, 1990, ms., p. 23 sq.

16. Cf. "La tenue d'un fichier domiciliaire: une obligation pour les communes d'AlsaceMoselle?», Revue du Droit local, 11, 1994, p. 27-30.

17. À Strasbourg, seules subsistent, aux archives départementales du Bas-Rhin, les listes nominatives du recensement de 1936. On trouve également quelques registres pour 1931 aux archives municipales.

18. Cf. S. Mathevon, Les élites strasbourgeoises de l'entre-deux-guerres, mémoire de DEA, Université de Strasbourg II, 1993, ms.

19. Cf. J.-L. Pinol, «Itinéraires résidentiels des universitaires strasbourgeois », Annales de la Recherche urbaine, 62-63, 1994, p. 157-168.

20. Sur les raisons de ce choix voir F.-J. Hahn et J.-L. Pinol, « La mobilité d'une grande ville: Strasbourg de 1870 à 1940 (présentation de l'enquête et premiers résultats) », Annales de Démographie historique, 1995, p. 197-210, qui présente également la base de données constituée.

21. Les zones géographiques sont définies en fonction des frontières des années trente, même si le pays n'existait pas en tant que tel avant la Première Guerre mondiale. La région Lorraine correspond à la partie annexée par le deuxième Reich. Sur ce point, voir ibid., p. 202.

22. À Lyon, à la veille de la Première Guerre mondiale, $25 \%$ des hommes adultes vivent dans la ville depuis plus de 15 ans. Voir J.-L. Pinol, Les mobilités de la grande ville..., op. cit., p. 219. À Lyon, les itinéraires ont été reconstitués à partir d'observations faites de cinq ans en cinq ans. À Strasbourg, l'observation est continue. On peut donc définir les enracinés par des durées de séjour variables.

23. Dans la première coupe, les chefs de ménage considérés comme enracinés résident à Strasbourg depuis 1874 au moins, année qui se situe après le départ des habitants qui ont opté pour la France après le traité de Francfort, alors que pour la seconde coupe, les chefs de ménage considérés comme enracinés résident à Strasbourg depuis 1909, c'est-à-dire que sont incluses les 
années de guerre et d'après-guerre qui ont enregistré de nombreux départs et l'expulsion, en 1919 et 1920, de nombreux Allemands.

24. Pour le dépouillement des fiches, l'adresse précise des habitants a été enregistrée mais le codage a été fait à partir d'un découpage élaboré par l'office statistique municipal. La ville a été divisée en 14 quartiers. Ces quartiers ont dans l'ensemble une assez forte unité morphologique. Sont prises en compte des lignes de fortifications, une partie des cours d'eau et des canaux qui séparent les diverses unités urbaines.

25. Cf. sur cet aspect K. Nohlen, Baupolitik im Reichsland Elsaß-Lothringen, 1871-1918. Die repräsentativen Staztsbauten um den ehemaligen Kaiserplatz in Straßburg, Berlin, Gebr. Mann Verlag, 1982.

26. Sur ce point, cf. F. Igersheim et J.-L. Pinol, «Strasbourg, la capitale du Reichsland» et «Strasbourg, une ville entre deux guerres », p. 134-137, in J.-L. Pinol, ed., Atlas historique des villes de France, Paris, Hachette, 1996.

27. Parmi les chefs de ménage qui restent moins de six mois à Strasbourg, $41 \%$ ont une seule adresse.

28. L'effectif indiqué ne correspond pas toujours à celui sur lequel a été calculé le séjour moyen en raison de l'absence de la date exacte d'entrée ou de sortie de la première adresse. Par exemple, sur les 80 chefs de ménage qui se sont installés entre 1890 et 1899 dans la vieille ville est, 22 d'entre eux y ont eu leur première et leur seconde adresse, mais la durée moyenne du séjour a été calculée sur les 21 pour lesquels il était possible de le faire.

29. Cf. J.-L. Pinol, « Lectures transversales... », op. cit.

\section{RÉSUMÉS}

La recomposition permanente de la ville, et plus particulièrement de la grande ville, ne peut véritablement être mise en évidence par l'analyse d'une succession de coupes transversales. Ce type d'analyse interdit de comprendre les rythmes de la recomposition elle-même, faute de reconstituer les trajectoires des urbains. Les sources les plus adéquates à cette enquête sont celles qui relèvent de la logique des registres de population, tel celui de Strasbourg, ouvert en 1871.

L'enquête porte sur les chefs de ménage dont le patronyme commence par la lettre $\mathrm{N}(1,2 \%$ de la population totale). L'information la plus importante est la suivante : $40 \%$ d'entre eux restent dans la ville moins de six mois, pour un séjour moyen inférieur à deux mois. L'étude s'attache à préciser les rapports qui existent entre population enracinée et "oiseaux de passage ", ainsi que les déplacements intra-urbains des chefs de ménage. Deux types de quartiers fermés sont ainsi mis en évidence: le premier correspond au nouveau quartier résidentiel construit après l'annexion au deuxième Reich, le second renvoie aux quartiers périphériques et populaires situés au sud de la ville.

The permanent rewards of the city and particularly of the big city cannot really be revealed by the analysis of a succession of cross sections. This kind of analysis does not allow us to understand the rythms of recomposition themselves because of the failure to reconstitute town dwellers trajectories. The most suitable sources for this inquiry are those which draw attention to the logic of population registers such as those of Strasburg, opened in 1871.

The inquiry deals with the heads of households whose surname begins with the letter $\mathrm{N}(1,2 \%$ of 
the population). The most significant evidence is that $40 \%$ of them remain in the city for less than six months for an average stay inferior to two months. The study applies itself to specifying the relationship between an established population and "migratory birds" as well as intra-urban movements of household heads. Two types of closed neighbourhoods are thus revealed: the first corresponds to the new residential neighbourhood constructed after annexation to the Second Reich; the second refers to the peripheral and popular neighbourhoods to the south of the city.

\section{AUTEUR}

\section{JEAN-LUC PINOL}

Jean-Luc Pinol (Université des Sciences humaines, Strasbourg) est spécialiste d'histoire urbaine contemporaine. Il a publié Le monde des villes au XIX ${ }^{e}$ siècle, Paris, Hachette, 1991 ; Les mobilités de la grande ville, Lyon fin XIX ${ }^{e}$-début XXe, Paris, Presses de la FNSP, 1991. Avec A. Zysberg, il a publié Métier d'historien avec un ordinateur, Paris, Nathan, 1995. Il vient de diriger l'Atlas historique des villes de France, Paris, Hachette, 1996. 